\title{
Metapopulation dynamics of the bog fritillary butterfly: modelling the effect of habitat fragmentation
}

\author{
Javier Sawchik ${ }^{\text {a, b,*, }}$, Marc Dufrêne a, c, Philippe Lebrun ${ }^{\text {a }}$, Nicolas Schtickzelle ${ }^{\text {a }}$, \\ Michel Baguette ${ }^{\text {a }}$ \\ ${ }^{a}$ Biodiversity Research Centre, Université catholique de Louvain, Belgium \\ b Sección Ecología Terrestre, Facultad de Ciencias, Universidad de la República, Iguá 4225, 11400 Montevideo, Uruguay \\ ${ }^{\mathrm{c}}$ Ministère de la Région Wallonne, Centre de Recherche de la Nature, des Forêts et du Bois, Observatoire de la Faune, de la Flore et des Habitats, \\ Belgium
}

Received 26 November 2001; accepted 29 April 2002

\begin{abstract}
Population viability analysis (PVA) and metapopulation theory are valuable tools to model the dynamics of spatially structured populations. In this article we used a spatially realistic population dynamic model to simulate the trajectory of a Proclossiana eunomia metapopulation in a network of habitat patches located in the Belgian Ardenne. Sensitivity analysis was used to evaluate the relative influence of the different parameters on the model output. We simulated habitat loss by removing a percentage of the original habitat, proportionally in each habitat patch. Additionally, we evaluated isolation and fragmentation effects by removing and dividing habitat patches from the network, respectively. The model predicted a slow decline of the metapopulation size and occupancy. Extinction risks predicted by the model were highly sensitive to environmental stochasticity and carrying capacity. For a determined level of habitat destruction, the expected lifetime of the metapopulation was highly dependent on the spatial configuration of the landscape. Moreover, when the proportion of removed habitat is above $40 \%$ of the original habitat, the loss of whole patches invariably leads to the strongest reduction in metapopulation viability. (C) 2002 Éditions scientifiques et médicales Elsevier SAS. All rights reserved.
\end{abstract}

Keywords: Spatially realistic models; Population dynamic models; Metapopulation dynamics; Isolation effect; Fragmentation

\section{Introduction}

Habitat loss and fragmentation induced by human activities are the main threats to biodiversity and species conservation (Wilcox and Murphy, 1985; Ehrlich, 1988). Habitat fragments are constantly reduced in their area and have progressively become more isolated. Consequently, local populations are restricted to small and isolated habitat patches and everyday more prone to extinction (Wilcove et al., 1986).

In particular, the landscape in Western Europe has been deeply modified by changes in agricultural practices during the 20th century that lead to the loss of semi-natural habitats

\footnotetext{
* Corresponding author.

E-mail address: sawchik@fcien.edu.uy (J. Sawchik).
}

and the progressive isolation of the remaining patches (Goffart et al., 2001). As a consequence, many species inhabiting such habitats are declining and show highly patchy distributions (Baguette and Goffart, 1991; Goffart et al., 1992). Hence, the rules of management focused on these habitats and species have to be derived in the frame of the metapopulation paradigm (Hanski, 1991; Hanski et al., 1996; Thomas and Hanski, 1997; Hanski, 1998b). A metapopulation is an ensemble of local populations connected by occasional migration events (Hanski, 1991; Hanski, 1998b). The inherent heterogeneous character of the environment determines that organisms are distributed in patches of favourable habitat (Gilpin, 1990). These local populations may have a high level of extinction risk. However, the movement of individuals among habitat patches allows the recolonisation of empty patches, decreas- 
ing the extinction risk of the ensemble (Hanski et al., 1996; Hanski, 1998b).

A fundamental step in the process of modelling metapopulation dynamics was the development of spatially realistic models (SRM), which incorporate specific information about spatial structure of the populations (Hanski, 1994; Hanski, 1997; Moilanen, 1998). SRM constitute an invaluable tool regarding population viability analysis (PVA), which has been developed to estimate the extinction likelihood of a population (Gilpin, 1990; Shaffer, 1990; Boyce, 1992; Burgman et al., 1996; Hanski, 1998b; Akçakaya and Sjögren-Gulve, 2000). Stochastic patch occupancy models (SPOMs) and population dynamic models (PDMs) are among the most frequently used types of SRMs. SPOMs assume that local population dynamics are much faster than regional dynamics. Therefore, these models ignore local dynamics and assume that metapopulation dynamics are determined only by extinction and colonisation processes (Hanski, 1994; Hanski, 1998b; Moilanen, 1998; Akçakaya and Sjögren-Gulve, 2000). Opposed to SPOMs are PDMs that simulate local dynamics and connect local populations through migration events (Hanski and Thomas, 1994; Burgman et al., 1996; Hanski, 1998b; Akçakaya, 2000; Akçakaya and Sjögren-Gulve, 2000). A disadvantage of these models is the great effort in parameterisation that they require (Hanski, 1998b; Akçakaya, 2000; Akçakaya and SjögrenGulve, 2000). However, they are more realistic and convenient than SPOMs for metapopulations, especially where species occupy nearly all patches (Hanski, 1994; Akçakaya and Sjögren-Gulve, 2000).

Butterflies have been proven to be suitable models to study spatially structured populations (Thomas and Hanski, 1997). Since they are easy to survey, they constitute an instrumentally tractable model. Moreover, many butterfly species are associated to well-defined habitats easy to locate within the landscape. Many specialist butterflies show a typical fragmented spatial structure in the landscape (Thomas and Hanski, 1997), and are declining in industrialised countries (Thomas, 1984). Hence, the long-run conservation of these species would be assured at the metapopulation level (Nève et al., 1996; Hanski, 1998a).

A primary goal of this study was to investigate how habitat loss and isolation should affect the persistence of Proclossiana eunomia populations within a spatially explicit landscape. The bog fritillary butterfly, P. eunomia, is a well-studied threatened species living in very localised habitat patches (Baguette and Goffart, 1991; Goffart and Waeyenbergh, 1994).

In particular, this study was aimed at: (1) the building of a SRM of the metapopulation dynamics of P. eunomia, parameterised with field data collected in a given network of habitat patches; (2) the identification of the most influent variables on the output model and the main threats for the persistence of the species; and (3) the assessment of the effect of habitat loss, fragmentation, and isolation on the system persistence by removing patches from the network.

\section{Materials and methods}

\subsection{The bog fritillary butterfly}

P. eunomia is a specialist butterfly species with a highly fragmented distribution in the southern part of its distribution range. In this region, it occurs in unfertilised wet meadows and peat bogs, where Polygonum bistorta, the only larval food plant and adult source of nectar, grows (Goffart and Waeyenbergh, 1994; Nève et al., 1996). Adults are on the wing in one generation from May to July, showing a marked protandry process (Schtickzelle et al., 2002). Females search actively for tussocks of certain gramineous (Deschampsia cespitosa, Molinia caerulea) to oviposit. Caterpillars use the straws of these plants for basking (Goffart and Waeyenbergh, 1994). Thus, their survivorship is not only dependent on the presence of the host plant but also on a determined vegetation structure.

\subsection{Study area}

The study area (ca. $20 \mathrm{~km}$ long, $15 \mathrm{~km}$ wide) is located in the Martin-Moulin, Aisne and Ourthe river basins. These river basins are situated in the 'Plateau des Tailles', Haute Ardenne, southern Belgium. Some very particular plant communities, like peat bogs and semi-natural meadows, develop due to the climatic and geomorphologic features of the region. In the landscape, they are more or less isolated in a matrix mainly constituted by anthropic habitats like spruce (Picea abies) plantations and fertilised pastures.

Thirty-two meadows were selected as potential habitat patches (mean area $=1$ ha, median area $=0.75$ ha, range 0.25-3 ha) for P. eunomia. The structure of the habitat patch network is detailed in Fig. 1.

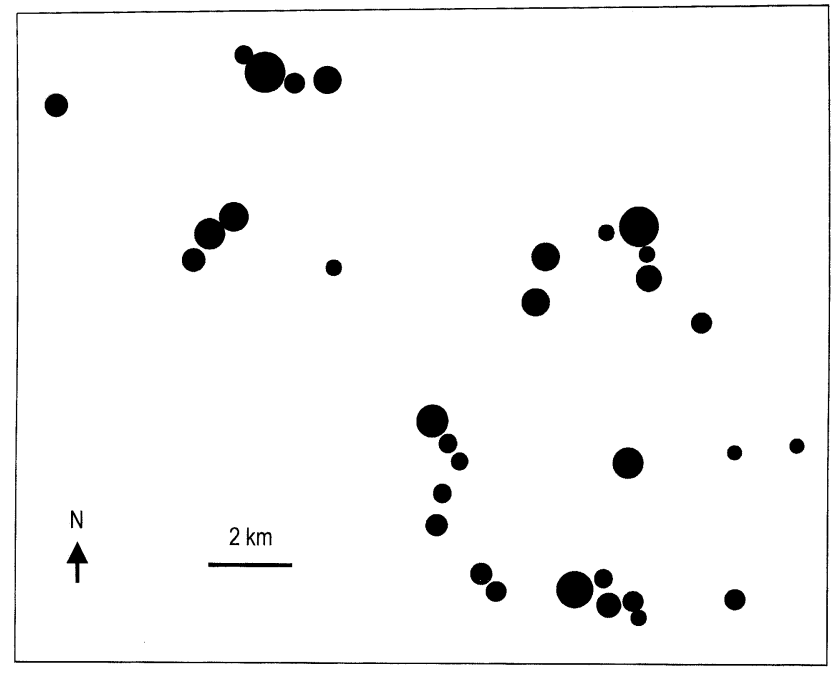

Fig. 1. The habitat patch network occupied by the bog fritillary in the Plateau des Tailles. Circle area is proportional to the estimated carrying capacity of the patch, which depends on the area and the habitat quality. 


\subsection{Sampling}

We recorded butterflies at each site, during the flight season (May-July) between 1994 and 1996. Counts were made every week by visual observation along transects, during fine weather conditions (Pollard, 1997; Thomas, 1983).

We used the maximum number of individuals observed in a day as an index of relative abundance. This measure was significantly correlated with population size, estimated by mark-release-recapture (MRR) studies (Baguette and Nève, 1994; Nève et al., 1996). Hence, we computed abundance at each site, by linear regression $\left(R^{2}=0.923\right.$; $p=0.026)$.

\subsection{Model description and parameterisation}

We constructed a female-only, discrete-time, spatially explicit, stochastic model to simulate the dynamics of a system constituted by 32 local populations. Local dynamics were modelled assuming a scramble-type densitydependence model, by using the Ricker function. Discrete time logistic models, as the Ricker function, have already been used to describe population growth in other species of butterflies (Hanski and Thomas, 1994; Hanski et al., 1994). The use of the Ricker function is justified in univoltine insects (Heino, 1998). Besides, scramble-type intraspecific competition may be suitable to describe situations where parasitism constitutes a key factor (Warren, 1992; Hanski, 1998b). This may be the case of P. eunomia, since this species has gregarious caterpillars, and more than $90 \%$ of the 4 th stage larvae collected in the field were parasitised by an hymenopteran Braconidae (Waeyenbergh and Baguette, 1996). As an additional advantage, scramble competition, compared to contest competition, generates conservative (higher) estimates of extinction probabilities.

Hanski and Thomas (1994) suggested values of 1.5 and 2 for the intrinsic rate of population increase $(r)$ for three butterfly species. In the present study, the intrinsic growth rate was fixed at the conservative value of 1.5 . This value corresponds to an approximately five-fold increase per year $(R=4.8) . R$ is the maximum proportion at which the population increases per year when there are no densitydependence effects. The growth rate diminishes when the population increases and approaches the carrying capacity (Akçakaya, 1994).

We specified the butterfly abundances estimated for the year 1994 as the initial starting populations. Data from years 1994-1996 were pooled to avoid annual weather effects, as well as local extinction events, and used to compute habitat quality at each patch. Measures of habitat quality were based on floristic composition (Sawchik, 1999). Carrying capacities were estimated with multiple regression using patch area and habitat quality (based on floristic composition) as predictor variables. The model, detailed in Sawchik (submitted for publication), explained $66 \%$ of the butterfly data variation. The average carrying capacity was 90 females per ha. This value was conservative compared with those suggested by Hanski and Thomas (1994).

We modelled environmental stochasticity by sampling the set of carrying capacities from log-normal random distributions with standard deviations (S.D.) taken as 50\% of the average values (Akçakaya, 1994). These random variations introduced in carrying capacities may generate high fluctuations that affect populations of any size (Heino, 1998). Environmental stochasticity was modelled without temporal autocorrelation, i.e. as a white noise.

A relationship between the synchrony level of local population dynamics and the inter-patch distance has been detected for several species, including butterflies and moth species (Sutcliffe et al., 1996). We assumed spatially autocorrelated environmental variability, with spatial correlation $\left(c_{i j}\right)$ inversely proportional to inter-patch distance. Thus, spatial correlation among local population dynamics was modelled fitting a negative exponential function:

$$
c_{i j}=a_{\mathrm{c}} \cdot \exp \left(\frac{-d_{i, j}^{c_{\mathrm{c}}}}{b_{\mathrm{c}}}\right),
$$

where $d_{i, j}$ is the distance between the patches $i$ and $j$, and $a_{\mathrm{c}}$, $b_{\mathrm{c}}$ and $c_{\mathrm{c}}$ are function parameters (Akçakaya, 1994). Increments in $a_{\mathrm{c}}$ and $b_{\mathrm{c}}$ increase spatial correlation $\left(c_{i j}\right)$, leading to highest levels of synchrony among local dynamics. Increments in $c_{\mathrm{c}}$ lead to a decrease of $c_{i j}$, lowering the level of synchrony. The parameters of the correlation function, were fixed arbitrarily $\left(a_{\mathrm{c}}=1 ; b_{\mathrm{c}}=10 ; c_{\mathrm{c}}=1\right)$. These values determined a correlation of $r \approx 0.98$ among the next habitat patches $(<1 \mathrm{~km})$ and of $r \approx 0.22$ among the most distant habitat patches $(>15 \mathrm{~km})$, which are plausible correlation values (Sutcliffe et al., 1996).

The distributions of distances travelled by individuals among habitat patches was modelled with a negative exponential function:

$$
m_{i j}=a_{\mathrm{m}} \cdot \exp \left(\frac{-d_{i, j}^{c_{\mathrm{m}}}}{b_{\mathrm{m}}}\right),
$$

where $d_{i, j}$ is the distance between the patches $i$ and $j$, and $a_{\mathrm{m}}$, $b_{\mathrm{m}}$ and $c_{\mathrm{m}}$ are function parameters (Akçakaya, 1994). As a result, migration was exclusively dependent on the interpatch distance. Negative exponential functions are commonly used to describe distribution of migration distances with good results, especially for organisms moving between discrete habitat patches (Baguette et al., 2000; Hill et al., 1996; Akçakaya et al., 1995; Akçakaya and Atwood, 1997). The parameter $a_{\mathrm{m}}$ corresponds to the migration rate, that is the proportion of emigrant individuals per time step from one population to another, and $b_{\mathrm{m}}$ represents the average distance an emigrant travels (Akçakaya, 1994). An effect of the patch area on the migration rates has been demonstrated by Hill et al. (1996). However, recent studies indicate that 
this effect is not always as simple as it was supposed (Baguette et al., 2000). Indeed, it was recently demonstrated that habitat quality could compensate for losses in habitat quantity (Kuussaari et al., 1996; Summerville and Christ, 2001; Thomas et al., 2001). Nevertheless, the fact that the patches with larger population size produced more emigrants than those with smaller population size has been clearly proved (Hill et al., 1996; Baguette et al., 2000). For these reasons, we preferred to use a simple approach based on the fitted negative exponential function, assuming patch area-independent migration rates. With this approach, the actual number of emigrants is a function of the migration rate and of the population size, in the source population. Mortality during migration is not explicitly modelled by the RAMAS package, assuming that it is accounted by local mortality (Akçakaya, 1994). Indeed, it seems that P. eunomia shows low cost of migration, particularly when the level of spatial isolation is low compared to the flight capacity of the species (Petit et al., 2001). In summary, the negative exponential function was fitted to the observed distribution of migration distances on data provided by extensive MRR studies (Baguette and Nève, 1994; Nève et al., 1996; Schtickzelle, 1997). Migration parameters $\left(a_{\mathrm{m}}=0.45\right.$ $\left.b_{\mathrm{m}}=0.71 ; \quad c_{\mathrm{m}}=1.00\right) \quad$ were estimated by maximumlikelihood, non-linear regression $\left(R^{2}=0.70 ; p=0.0001\right)$.

Demographic stochasticity and Allee effects were not modelled explicitly to gain in simplicity and simulation time.

\subsection{Sensitivity analysis}

Sensitivity analysis is a useful method to evaluate how sensitive the model response is to the variation of each input parameter (Burgman et al., 1996; Akçakaya, 2000). Thus, sensitivity coefficients can be used to evaluate the potential model error, to direct future research on parameter estimation, and to rank potential management options (Burgman et al., 1996; Drechsler, 1998; Drechsler et al., 1998; Akçakaya, 2000).

For the present study, we selected the extinction risk $(Q)$ as the state variable. Sensitivity coefficients were estimated controlling the variation of an individual parameter $(\Delta p / p)$ and checking the proportional change in the value of the model output or state variable $(\Delta Q / Q)$ (Burgman et al., 1996). Sensitivity coefficients were computed as:

$$
C_{p}=-\frac{\operatorname{logit} Q(p+\delta p)-\operatorname{logit} Q(p)}{\delta},
$$

where $C_{P}$ is the sensitivity coefficient that quantifies the influence of varying the parameter, $p$, by a relative proportion, $\delta$, on the extinction risk (Drechsler et al., 1998). We computed sensitivity coefficients for the dispersion function parameters $\left(a_{\mathrm{m}}, b_{\mathrm{m}}, c_{\mathrm{m}}\right)$, the correlation function parameters $\left(b_{\mathrm{c}}, c_{\mathrm{c}}\right)$, the growth rate and the mean and S.D. of the carrying capacity. Each parameter was varied in turn by a proportion of $\delta=( \pm 10,30$ and $50 \%)$.

\subsection{Habitat loss and fragmentation}

There is no single ideal method to describe the degree of isolation of the patches (Hanski and Thomas, 1994; Clinchy et al., 2002). To measure the degree of isolation of each patch, we used a simple index computed as the average distance between a patch and other occupied patches (Hanski et al., 1994). Pearson's product-moment correlation coefficients were computed between local occupancy, isolation and the carrying capacity of the habitat patches.

We simulated habitat loss by removing a percentage (10, $20,30,40,50$ and $60 \%$ ) of total carrying capacity (sum of carrying capacity over all habitat patches). We constructed four 'artificial landscapes' representing different degrees of connectivity. The first scenario was obtained by reducing a percentage of total carrying capacity, proportionally in each habitat patch. Habitat loss results in a constant number of smaller patches. Scenarios II and III were constructed by removing the same percentage of habitat, but as whole patches; thus, the number of patches was reduced. In scenario II we removed the habitat patches with largest $K$. In general, these patches are the largest because $K$ is a function of the area and the habitat quality of the patches, so this scenario resulted in a fine-grain landscape (Rolstod, 1997). Removal of the patches with smallest $K$ (scenario III) arose in a coarse-grain landscape. These different artificial landscapes, with equal total carrying capacity, showed different degrees of increasing overall isolation.

A fundamental issue is to distinguish fragmentation from habitat loss effects (Fahrig and Merriam, 1994; Fahrig, 1997). These processes occur together and their effects on population extinction are confounded. Fragmentation occurs only when the number of habitat patches increases as a consequence of the division of original patches in separate sections (Fahrig, 1997). We simulated fragmentation by modifying scenario I, choosing the eight habitat patches with the largest $K$ and dividing each of them in two almost contiguous patches of smaller $K$ compared to the original patch. In this way, the total number of habitat patches increased to 40 (scenario IV). Because the new habitat patches created from an original patch are very close to each other, migration and correlation values between them are maximum.

Simulations were carried out with the program RAMAS/METAPOP (Akçakaya, 1994). The model was run for each scenario and projected for 50 years. All simulations were run with 1000 replications. The significance level of the maximum distance among the extinction curves, obtained for the different scenarios, was assessed by Kolmogorov-Smirnov tests (Akçakaya, 1994). 


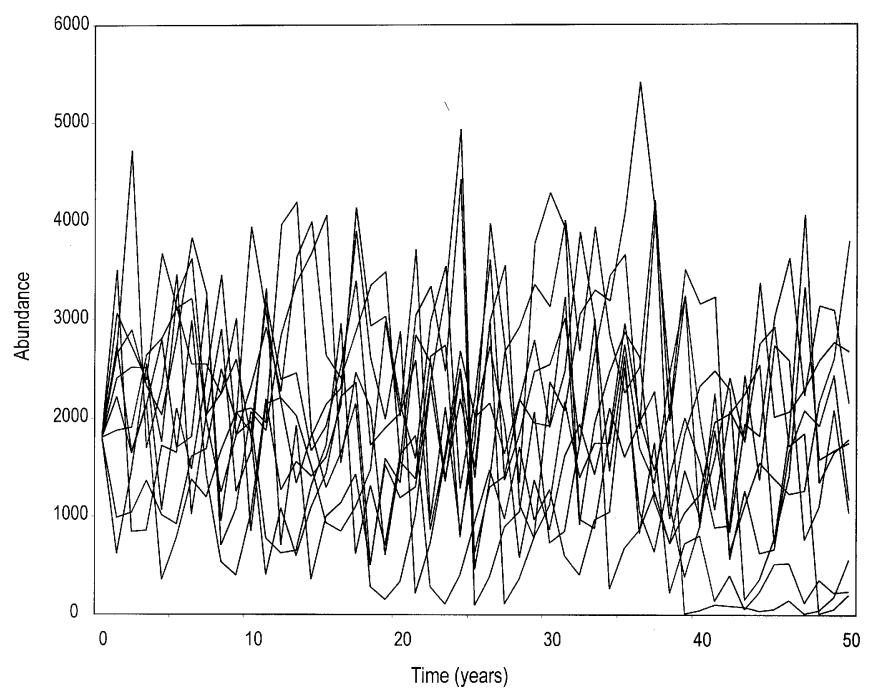

Fig. 2. Metapopulation trajectory over 50 years in 10 replicate simulations.

\section{Results}

\subsection{Model predictions}

Both, the average number of individuals and the average number of occupied patches showed a slight declining trend. Fig. 2 shows 10 simulated metapopulation trajectories produced by the model. The predicted extinction risk of the metapopulation was of $2.4 \%$ over 50 years. An extinction risk of $0.5 \%$ over 50 years was predicted when we ran the model incorporating contest competition. Local occupancy (number of years that a patch remains occupied) was negatively correlated with isolation $(r=-0.858, p<0.001)$. We did not find a significant correlation between local occupancy and carrying capacity.

\subsection{Sensitivity analysis}

Sensitivity coefficients are presented in Table 1. A positive sensitivity coefficient denotes that the extinction risk declines with an increase in the parameter considered.

The S.D. and the mean of carrying capacities were the parameters that showed the highest sensitivity coefficients for all the considered variation levels. In decreasing order, the parameters that showed the following sensitivity coefficients were those that determine the correlation level between local dynamics $\left(b_{\mathrm{c}}\right.$ and $c_{\mathrm{c}}$ ) and those that define the migration power of the species $\left(b_{\mathrm{m}}\right.$ and $\left.c_{\mathrm{m}}\right)$. The growth rate was clearly the parameter to whose variations the model was less sensitive.

The average carrying capacity, the parameters $a_{\mathrm{m}}$ and $b_{\mathrm{m}}$ of the dispersion function, and the parameter $c_{\mathrm{c}}$ of the correlation function showed positive sensitivity coefficients to positive increments. Thus, increasing the value of these parameters leads to a decrease in the extinction risk. Negative sensitivity coefficients were found for the S.D. of carrying capacity, the parameter $c_{\mathrm{m}}$ of the dispersion function and the parameter $b_{c}$ of the correlation function. When increasing the value of these parameters there is an increment of the extinction risk.

\subsection{Habitat loss}

Extinction risk curves for the different scenarios tested are shown in Fig. 3(a-f). Significant differences among most of the probability curves were detected by means of Kolgomorov-Smirnov tests Fig. 3(a-f). Removal of whole habitat patches (scenarios II and III) led to a greater

Table 1

Sensitivity coefficients for the extinction risk to different variations (expressed as percentages) of the model parameters (mean carrying capacity, $K$; standard deviation of the carrying capacity, $K$-std; growth rate, $R$ )

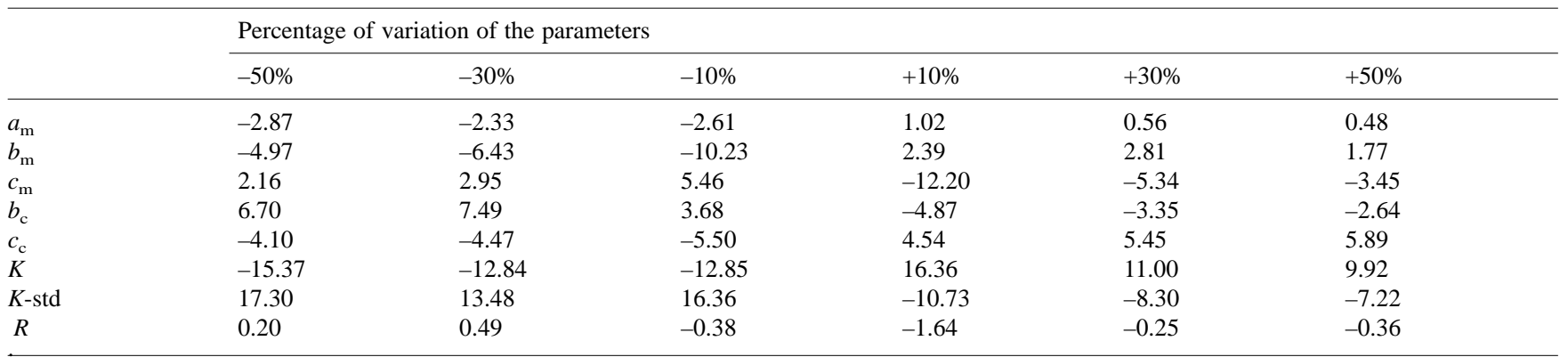



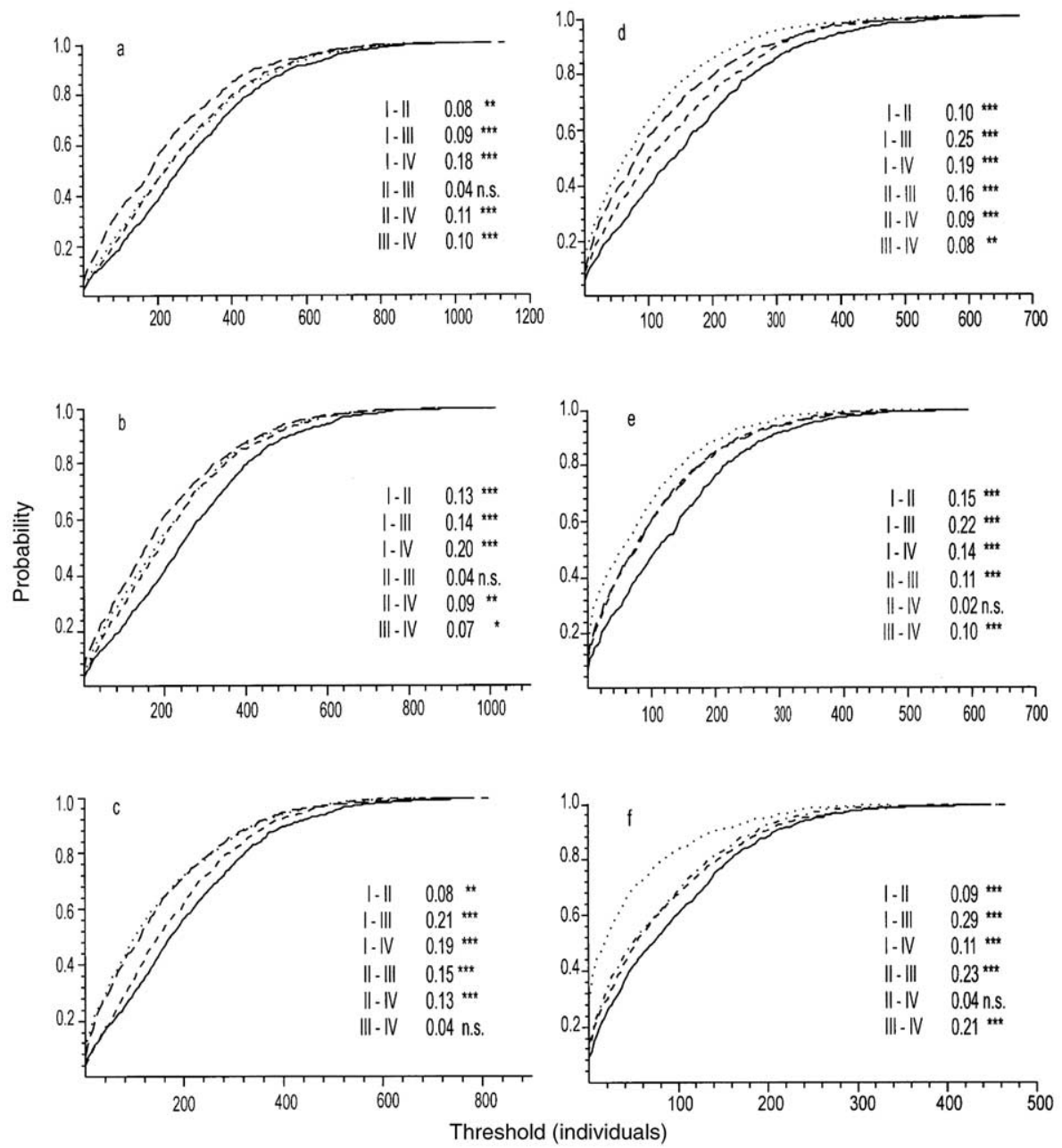

Fig. 3. Extinction risk curves of the different scenarios (scenario I: solid line; scenario II: dotted line; scenario III: dashed line; scenario IV: broken line). The asterisks give the significance level of the Kolmogorov-Smirnov statistic computed to test the difference (maximum vertical distance) between the curves $(0.05 *, 0.01 * *, 0.001 * * *)$. The carrying capacity of the metapopulation was lowered by 10 (a), 20 (b), 30 (c), 40 (d), 50 (e) and $60 \%$ (f).

extinction risk than the proportional reduction of carrying capacity in all patches (scenario I). Removal of largest patches (scenario II) had a lower effect on the extinction risk than removal of smallest patches (scenario III): the extinction probability was higher in the second case (Table 2). This pattern may be observed for all the levels of variation imposed on the system Fig. 3(a-f).

Compared to scenario I, fragmentation (scenario IV) always increased the extinction risk of the system. At low and medium levels of habitat loss (10-40\%), scenario IV led to higher estimated extinction risks than those obtained with scenario II. There were not significant differences between scenarios II and IV, if habitat loss was from 50 to $60 \%$. A more complex pattern can be discerned when we compare scenario IV with to scenario III. At low levels of habitat loss (10-20\%), scenario III showed lower extinction risks than those obtained with scenario IV. This situation is inverted when the level of habitat loss increases. When there was $40 \%$ or more of habitat loss, the probability of extinction was significantly larger in scenario III.

\section{Table 2}

Metapopulation extinction risk after removal of a percentage of total $K$. Four scenarios were tested (scenario I: $K$ reduced proportionally for all patches; scenario II: removal of the largest patches; scenario III: removal of the smallest patches; scenario IV: fragmented patches)

\begin{tabular}{lllllrr}
\hline \multicolumn{7}{l}{ Proportion of the total habitat $(K)$ removed } \\
\cline { 2 - 7 } & $-50 \%$ & $-30 \%$ & $-10 \%$ & $+10 \%$ & $+30 \%$ & $+50 \%$ \\
\hline I & 2.4 & 2.9 & 4.2 & 5.5 & 6.3 & 9.1 \\
II & 2.9 & 3.0 & 3.9 & 8.5 & 10.6 & 14.2 \\
III & 3.4 & 4.1 & 9.6 & 13.5 & 16.5 & 28.9 \\
IV & 5.5 & 5.8 & 7.2 & 9.2 & 11.1 & 12.3 \\
\hline
\end{tabular}




\section{Discussion}

\subsection{Model predictions}

The declining trend of the system predicted by the model seems to be in agreement with the situation registered in Belgium, in which a decrease in the number of individuals and of occupied patches has been reported (Baguette et al., 1992).

In the prospected basins, P. eunomia showed the tendency to occupy most of the habitat patches during the period of study (3 years). In this period, only two extinction events were registered. Moreover, these extinctions occurred in habitat patches of low quality, one of them suffering a progressive invasion by Urtica dioica (Sawchik, 1999; Goffart et al., 2001). Thus, the metapopulation seems to be stable at a short time scale. Model predictions may conform to this pattern, considering the slow declining trend of the system trajectory and the short-time of the study period. The short-time viability of the metapopulation is probably assured by a network of abundant habitat patches and by the good migration power of the species (Baguette and Nève, 1994; Nève et al., 1996; Petit et al., 2001).

Model predictions have to be taken cautiously because some potential causes of local extinction (genetic and demographic stochasticity, social dysfunction) were not considered. However, these factors are really important only on small-size populations (Shaffer 1990; Hanski 1991; Hanski and Thomas 1994; Thomas and Hanski, 1997). The effect will mainly be on satellite patches with poor quality and/or small area, e.g. sinks (Watkinson and Sutherland, 1995). Thus, it is uncertain that these factors affect large habitat patches, which are the main sources of individuals (Harrison and Taylor, 1997). Moreover, experiments that were carried out in the Morvan region (France) showed that P. eunomia has a strong colonisation capability at local scale. Starting from a few introduced females, the population increased considerably and expanded in a new region (Nève et al. 1996; Barascud et al., 1999). We supposed that if small-population factors like Allee effects had been particularly prominent, these populations would have not prospered.

The assumption of a binary landscape with an isotropic inter-patch matrix was not a restrictive premise since P. eunomia is distributed in well-defined habitat patches and shows a high migration power (Nève et al., 1996). It has been observed that this species is able to cross unfavourable and unattractive habitats, as coniferous forests and cultivated prairies (Nève et al., 1996). Movements of individuals have also been observed between two valleys, and even two different oro-hydrographic basins (Nève et al., 1996; Goffart et al., 2001; Petit et al., 2001). On the other hand, some assumptions could lead to a pessimistic behaviour of the model. For instance, the negative exponential function used to model migration may lead to underestimates of the long-distances migration probability (Hill et al., 1996; Schtickzelle, 1997; Baguette et al., 2000). Lastly, some local populations identified in other oro-hydrographic basins were not included in the analysis. As was previously stated, we have recorded exchanges of individuals across valleys and even among different basins. Consequently, it is probable that the metapopulation is constituted by more habitat patches than those considered, buffering its chances of extinction (Hanski, 1998a). Thus, our model is a trade-off between pessimistic and optimistic simplifications.

\subsection{Sensitivity analysis}

The high sensitivity coefficients of the S.D. of carrying capacities and of the correlation parameters showed that the model was strongly sensitive to regional stochasticity, i.e. spatially correlated environmental stochasticity (Hanski, 1991; Hanski 1997). Increases in regional stochasticity, given by increments in the environmental stochasticity and/or the correlation between local dynamics, determine an increase in the extinction risk of the metapopulation. Unfortunately, empirically quantifying the impact of regional stochasticity is very difficult, and most studies are of too short duration to obtain reliable estimates of spatio-temporal variation of the vital rates or the habitat (Shaffer, 1990; Burgman et al., 1996; Hanski, 1998b; Brooks, 2000).

The carrying capacity of the habitat patches had a strong influence on the model output. This fact highlights the importance that local conditions, i.e. habitat quality and quantity, have on local extinction and metapopulation viability (Harrison and Taylor, 1997; Drechsler and Wissel, 1998; Etienne and Heeterbeek, 2001). SPOMs ignore local dynamics, and are traditionally biased towards the analysis of area and connectivity effects despite habitat quality. However, recent studies have shown that habitat quality may be an efficient predictor variable of the species occupancy, even more than the traditionally used by the metapopulation approach (Summerville and Crist, 2001; Thomas et al., 2001). For these reasons, PDMs may constitute one of the most valuable option for PVA, especially when local dynamics and habitat quality seem to play an important role in metapopulation persistence. PDMs are more suitable than SPOMs also in the case of metapopulations that occupy most of the habitat patches in a network and/or when low levels of turnover events are detected (Akçakaya and Sjögren-Gulve, 2000). Moreover, PDMs are more flexible than SPOMs and, in the case of well-studied species, they allow the development of demographically structured models that may greatly improve the quality of the results (Akçakaya, 2000; Akçakaya and Sjögren-Gulve, 2000). These properties make PDMs an interesting alternative approach to model butterfly metapopulations, especially when we are interested in metapopulation viability in a determined patch network.

Extinction risk was also very sensitive to variations in $b_{\mathrm{m}}$ and $c_{\mathrm{m}}$ (migration parameters), indicating that the average and the maximum migration distance have much influence on the persistence of the system than the maximum migration rate (Baguette and Nève, 1994; Nève et al., 1996). 
Finally, the model was reasonably robust to the variations of the growth rate and then, no great efforts would be made to obtain very accurate estimates.

\subsection{Habitat loss and isolation effect}

The explicit inclusion of spatial structure in models has been one of the last great advances in theoretical ecology (Gilpin, 1990; Dunning et al., 1995; Burgman et al., 1996; Hanski 1998a). Hence, the central role of spatial structure in the metapopulation life expectancy, has been demonstrated (Hill et al., 1996; Moilanen et al., 1998; Baguette et al., 2000). Our results suggest that the removal of whole patches could have stronger detrimental effects on the metapopulation life expectancy than the reduction of habitat quantity on each patch. Extinction risk was generally higher when we removed whole habitat patches, that is to say, when the number of habitat patches was reduced. The remnant habitat patches turned progressively more isolated as a consequence of the decrease in the number of patches. Then, vacant patches became less susceptible to be colonised and local populations showed low chances to receive immigrants that might reduce the extinction risk via the rescue effect (Hanski et al., 1995; Hanski et al., 1994).

The fragmentation effect has not always been separated from the single effect produced by habitat reduction (Fahrig, 1997). Our simulations suggest that habitat fragmentation per se has adverse effects on metapopulation dynamics. The extinction risk of the metapopulation in a non-fragmented habitat (scenario I) was lower than in a fragmented one (scenario IV). However, more complex patterns were obtained when compared with scenarios that have lost entire habitat patches. For moderate levels of habitat loss, fragmentation may lead to significant decreases in metapopulation life expectancy. However, when the level of habitat loss increases, the effect of the loss of whole habitat patches becomes progressively more intense and exceeds the fragmentation effect.

Our results show the fundamental importance that landscape physiognomy and isolation, i.e. the number and spatial configuration of habitat patches, have on the expected metapopulation lifetime. The significance of isolation was also manifested by the fact that the habitat patches with the lowest predicted local occupancy were the most isolated. These patches have probably low chances of recolonisation if they become extinct. Moreover, local occupancy of the best connected patches may be enhanced by rescue effects (Hanski, 1998b; Hanski et al., 1994; Moilanen, 1998).

These conclusions are opposed to those obtained in a recent study conducted by Hanski and Ovaskainen (2000). These authors found that decreasing areas of the habitat patches was often more harmful than loss of entire patches. Evidently, the results may greatly vary depending on the combined effect of various factors such as the level of regional stochasticity, the explicit configuration of the landscape, the dispersal behaviour of the species, and the quality of the habitat patches (Fahrig and Merriam, 1994; Hanski et al., 1996; Hanski 1998a). Thus, when we compare different studies we must be extremely cautious because each case reflects the particular characteristics of the species and a certain landscape physiognomy. Additionally, we must also consider that non-linear responses of the model to the variation of the parameters could lead to more complex dynamics.

\subsection{Conservation considerations}

It is possible that many favourable habitat patches for P. eunomia have been suffering a deterioration of their carrying capacity. First, these habitats are of transient nature and so successional changes lead to the colonisation by woody species (Goffart and Waeyenbergh, 1994; Nève et al., 1996). This process has been enhanced by changes in agricultural practises. Second, habitat loss and fragmentation have increased due to plantations of the spruce. Finally, the enrichment with nutrients of anthropogenic origin leads to the progressive colonisation of nitrophyllous and very invasive species like Filipendula ulmaria and $U$. dioica (Sawchik, 1999; Goffart et al., 2001). These species can competitively exclude P. bistorta and deeply modify the structure of the vegetation needed for the persistence of $P$. eunomia populations. Thus, it is possible that $P$. eunomia was threatened by deterministic pressures, i.e. habitat loss induced by succession and disturbances (Harrison and Taylor, 1997). Then, the most efficient action to preserve these populations is to stop the processes that lead to habitat destruction and degradation (Hanski, 1998a).

A fundamental point is to preserve suitable habitat by conserving sites with high carrying capacity as potential refuges. These sites are the main sources of individuals and have a crucial value assuring the recolonisation of empty patches (Harrison, 1991; Thomas and Hanski, 1997). It is also important to protect the main sites from habitat changes that could increase the variability in their carrying capacities (Ruggiero et al., 1994). The selection of a set of sites that represent a broad range in habitat quality may help to buffer the adverse effects of regional stochasticity (Hanski, 1997; Thomas and Hanski, 1997; Hanski, 1998a).

A network of abundant habitat patches may enhance the persistence of the metapopulation (Hanski et al., 1995). This 'exuberant' network allows the flow of individuals among habitat patches assuring the turnover between stochastic local extinctions and recolonisations. Hence, the preservation of a high number of habitat patches is an important step in order to assure the metapopulation viability. Spatial configuration of habitat patches is particularly important for regional dynamics and must be considered on management plans (Dunning et al., 1995). Depending on their spatial attributes, some small patches may have an essential role acting as a stepping-stones (Gilpin, 1980; Shaffer 1990; Nève et al., 1996; Moilanen et al., 1998), and therefore, important efforts must be made to identify, restore and preserve these patches. 


\section{Conclusion}

The model predicted that the $P$. eunomia populations may be slowly declining and thus, their persistence would not be assured at long term. The metapopulation persists at shorttime scale probably due to the presence of a network with abundant suitable habitat patches and the high migration power of the species. Nevertheless, an active management of suitable habitat may be indispensable to assure the survival of $P$. eunomia in the studied region. Probably, this only could be achieved by the interruption of the processes that promote habitat loss. To prevent the detrimental effect of colonisation-extinction stochasticity, the preservation of networks of many habitat patches is required (Hanski, 1991; Hanski et al., 1996). Particularly, the conservation of large and high-quality habitat patches, which may play a fundamental role as refuges, is crucial. Additionally, the restoration of small but well connected patches, which may perform as potential stepping-stones, should also be considered (Nève et al., 1996; Moilanen et al., 1998).

Regional stochasticity and carrying capacity have a strong effect on the metapopulation expected lifetime. Consequently, great efforts would have to be made to elucidate the impact of spatial and temporal environmental variation on the vital rates (Shaffer, 1990; Hanski, 1991; Thomas and Hanski, 1997).

PDMs are among the most objective approaches to metapopulation modelling and risk analysis. Nevertheless, predictions derived from PDMs studies must be carefully considered when applied to real problems of conservation biology. PVAs are species-specific (Baguette et al., 2000), model assumptions must be clearly exposed and model results must be always interpreted in particular spatial and temporal scales (Fahrig and Merriam, 1994; Hanski et al., 1996). Evidently, the outcome of the model strongly depends on the configuration of the landscape (number, area, location of the habitat patches). Moreover, the distribution and the quality of the habitat patches may change widely in a relatively short time, especially in transient or human disturbed habitats (Fahrig and Merriam, 1994). Furthermore, sensitivity analysis might be used to assess the relative importance of different parameters on the output of the model and to guide future fieldwork (Moilanen, 1998; Akçakaya and Sjögren-Gulve, 2000). PDMs are highly sensitive to the precision of the parameters inputted into the model. Given the uncertainty of several values used in the present paper, we can expect that parameter estimates using high-tech methods will provide less speculative conclusions.

\section{Acknowledgements}

We thank M. Alzate, C. Gilot and L. Wargé for butterfly samplings. L. Giménez, P. Goffart, G. Nève, N. Prigioni, M. Waeyenbergh, provide valuable inputs thorough the study process and on the first drafts of the manuscript. We would like to thank two anonymous referees for their helpful comments on the first version of this article. Financial support was provided by a Uruguyan subvention from DINACYT and a contract of the Ministère de la Région wallonne. This is contribution BRC009 from the Biodiversity Research Centre (UCL).

\section{References}

Akçakaya, H.R., 1994. RAMAS/GIS: linking landscape data with population viability analysis (version 1.0). Applied Biomathematics, New York.

Akçakaya, H.R., 2000. Population viability analysis with demographically and spatially structured models. Ecol. Bull. 48, 23-38.

Akçakaya, H.R., Atwood, J.L., 1997. A habitat-based metapopulation model of the California gnatcatcher. Conserv. Biol. 11, 422-434.

Akçakaya, H.R., Sjögren-Gulve, P., 2000. Population viability analyses in conservation planning: an overview. Ecol. Bull. 48, 9-21.

Akçakaya, H.R., McCarthy, M.A., Pearce, J.L., 1995. Linking landscape data with population viability analysis: management options for the helmeted honeyeater Lychenostomus melanops cassidix. Biol. Conserv. 73, 169-176.

Baguette, M., Goffart, P., 1991. Liste rouge des lépidoptères rhopalocères de Belgique. Bull. Annls. Soc. R. Belge. Entomol. 157, 147-153.

Baguette, M., Nève, G., 1994. Adult movements between populations in the specialist butterfly Proclossiana eunomia. Ecol. Entomol. 19, 1-5.

Baguette, M., Goffart, P., Bast de, B., 1992. Modification de la distribution et du statut des lepidoptères rhopalocères en Belgique depuis 1990. Mém. Soc. R. Belge. Entomol. 35, 591-596.

Baguette, M., Petit, S., Quéva, F., 2000. Population spatial structure and migration of three butterfly species within the same habitat network: consequences for conservation. J. Appl. Ecol. 37, 100-108.

Barascud, B., Martin, J.F., Baguette, M., Descimon, H., 1999. Genetic consequences of an introduction-colonization process in an endangered butterfly species. J. Evol. Biol. 12, 697-709.

Boyce, M.S., 1992. Population viability analysis. Annu. Rev. Ecol. Syst. 23, 481-506.

Brooks, B.W., 2000. Pessimistic and optimistic bias in population viability analysis. Conserv. Biol. 14, 564-566.

Burgman, M.A., Ferson, S., Akçakaya, H.R., 1996. Risk Assessment in Conservation Biology. Chapman \& Hall, London.

Clinchy, M., Haydon, D.T., Smith, A.T., 2002. Pattern does not equal process: what does patch occupancy really tell us about metapopulation dynamics? Am. Nat. 159, 351-362.

Drechsler, M., 1998. Sensitivity analysis of complex models. Biol. Conserv. 86, 401-412.

Drechsler, M., Wissel, C., 1998. Trade-offs between local and regional scale management of metapopulations. Biol. Conserv. 83, 31-41.

Drechsler, M., Burgman, M., Menkhorst, P.W., 1998. Uncertainty in population dynamics and its consequences for the management of the orange-bellied parrot Neophema chrysogaster. Biol. Conserv. 84, 269-281.

Dunning, J.B., Stewart, D.J., Danielson, B., Noon, B.R., Root, T.L., Lamberson, R.H., et al., 1995. Spatially explicit population models: current forms and future uses. Ecol. Appl. 5, 3-11.

Ehrlich, P.R., 1988. The loss of diversity: Causes and consequences. In: Wilson, E.O., Peter, F.M. (Eds.), Biodiversity. National Academy Press, Washington D.C, pp. 212-216.

Etienne, R.S., Heeterbeek, J.A.P., 2001. Rules of thumb for conservation of metapopulations based on a stochastic winking-patch model. Am. Nat. 158, 389-407.

Fahrig, L., 1997. Relative effects of habitat loss and fragmentation on population extinction. J. Wildl. Manage. 61, 603-610. 
Fahrig, L., Merriam, G., 1994. Conservation of fragmented populations. Conserv. Biol. 8, 50-59.

Gilpin, M.E., 1980. The role of stepping-stone islands. Theor. Popul. Biol. 17, 247-253.

Gilpin, M.E., 1990. Spatial structure and population vulnerability. In: Soulé, M.E. (Ed.), Viable Populations for Conservation. Cambridge University Press, Cambridge, pp. 125-140.

Goffart, P., Waeyenbergh, M., 1994. Exigences écologiques et gestion des populations de deux papillons des prairies humides ardennaises: le cuivré et le nacré de la bistorte (Lycaena helle, Proclossiana eunomia). Cah. Réserves Nat. 7, 21-29.

Goffart, P., Baguette, M., Bast De, B., 1992. La situation des lepidoptères rhopalocères en Wallonie ou Que sont nos papillons devenus? Bull. Soc. R. Belge. Entomol. 128, 355-392.

Goffart, P., Baguette, M., Dufrêne, M., Mousson, L., Nève, G., Sawchik, J., et al., 2001. Gestion des milieux semi-naturels et restauration de populations menacées de papillons de jour, Région wallonne, Direction Générale des Ressources naturelles de de l'Environnement, Travaux $n^{\circ} 25$, Jambes.

Hanski, I., 1991. Single-species metapopulation dynamics: concepts, models and observations. In: Gilpin, M.E., Hanski, I. (Eds.), Metapopulation Dynamics: Empirical and Theoretical Investigations. Academic Press, London, pp. 3-16.

Hanski, I., 1994. A practical model of metapopulation dynamics. J. Anim. Ecol. 63, 151-162.

Hanski, I., 1997. Metapopulation dynamics: from concepts and observations to predictive models. In: Hanski, I., Gilpin, M.E. (Eds.), Metapopulation Biology, Ecology, Genetics and Evolution. Academic Press, San Diego, pp. 69-91.

Hanski, I., 1998a. Metapopulation dynamics. Nature 396, 41-49.

Hanski, I., 1998b. Metapopulation Ecology. Oxford University Press, Oxford.

Hanski, I., Ovaskainen, O., 2000. The metapopulation capacity of a fragmented landscape. Nature 404, 755-758.

Hanski, I., Koussaari, M., Nieminen, M., 1997. Metapopulation structure and migration in the butterfly Melitaea cinxia. Ecology 75, 747-762.

Hanski, I., Thomas, C.D., 1994. Metapopulation dynamics and conservation: a spatially explicit model applied to butterflies. Biol. Conserv. 68, 167-180.

Hanski, I., Moilanen, A., Pakkala, T., Kuussaari, M., 1996. The quantitative incidence function model and persistence of an endangered butterfly metapopulation. Conserv. Biol. 10, 578-590.

Hanski, I., Pakkala, T., Kuussaari, M., Lei, G., 1995. Metapopulation persistence of an endangered butterfly in a fragmented landscape. Oikos 72, 21-28.

Harrison, S., 1991. Local extinction in a metapopulation context: an empirical evaluation. In: Gilpin, M.E., Hanski, I. (Eds.), Metapopulation Biology, Ecology, Genetics and Evolution. Academic Press, San Diego, pp. 73-88.

Harrison, S., Taylor, A.D., 1997. Empirical evidence for metapopulation dynamics. In: Hanski, I., Gilpin, M.E. (Eds.), Metapopulation Biology, Ecology, Genetics and Evolution. Academic Press, San Diego, pp. 27-42.

Heino, M., 1998. Noise colour, synchrony and extinctions in spatially structured populations. Oikos 83, 368-375.

Hill, J.K., Thomas, C.D., Lewis, O.T., 1996. Effects of habitat patch size and isolation on dispersal by Hesperia comma butterflies: implications for metapopulation structure. J. Anim. Ecol. 65, 725-735.

Kuussaari, M., Nieminen, M., Hanski, I., 1996. An experimental study in the Glanville fritillary butterfly Meliaea cinxia. J. Anim. Ecol. 65, 791-801.

Moilanen, A., 1998. Modeling metapopulation dynamics, Academic Dissertation. University of Helsinki, Helsinki.

Moilanen, A., Smith, A.T., Hanski, I., 1998. Long-term dynamics in a metapopulation of the American pika. Am. Nat. 152, 530-542.
Nève, G., Barascud, B., Hughes, R., Aubert, J., Descimon, H., Lebrun, P., et al., 1996. Dispersal, colonisation power and metapopulation structure in the vulnerable butterfly Proclossiana eunomia (Lepidoptera, Nymphalidae). J. Appl. Ecol. 33, 14-22.

Petit, S., Moilanen, A., Hanski, I., Baguette, M., 2001. Metapopulation dynamics of the bog fritillary butterfly: movements between habitat patches. Oikos 92, 491-500.

Pollard, E., 1997. A method of assessing changes in the abundance of butterflies. Biol. Conserv. 12, 115-134.

Rolstod, J., 1997. Consequences of forest fragmentation for the dynamics of bird populations: conceptual issues and the evidence. In: Hanski, I., Gilpin, M.E. (Eds.), Metapopulation Biology, Ecology, Genetics and Evolution. Academic Press, San Diego, pp. 149-163.

Ruggiero, L.F., Hayward, G.D., Squires, J.R., 1994. Viability analysis in biological evaluations: concepts of population viability analysis, biological population, and ecological scale. Conserv. Biol. 8, 364-372.

Sawchik, J., 1999. Répartition spatiale et dynamique de populations de rhopalocères des prairies humides au Plateau des Tailles, Ph.D. thesis, Université catholique de Louvain.

Schtickzelle, N., 1997. Variation des paramètres démographiques d'une population subdivisée du nacré de la bistorte Proclossiana eunomia (Lepidoptera, Nymphalidae), Mémoire de Licence, Université catholique de Louvain, Louvain-la-Neuve.

Schtickzelle, N., Le Boulengé, E., Baguette, M., 2002. Metapopulation dynamics of the bog fritillary butterfly: demographic processes in a patchy population. Oikos 97, 349-360.

Shaffer, M.L., 1990. Minimum viable population: coping with uncertainty. In: Soulé, M.E. (Ed.), Viable Populations for Conservation. Cambridge University Press, Cambridge, pp. 69-86.

Summerville, K.S., Christ, T.O., 2001. Effects of experimental habitat fragmentation on patch use by butterflies and skippers (Lepidoptera). Ecology 82, 1360-1370.

Sutcliffe, O.L., Thomas, C.D., Moss, D., 1996. Spatial synchrony and asynchrony in butterfly population dynamics. J. Anim. Ecol. 65, 85-95.

Thomas, C.D., Hanski, I., 1997. Butterfly metapopulations. In: Hanski, I., Gilpin, M.E. (Eds.), Metapopulation Biology, Ecology, Genetics and Evolution. Academic Press, San Diego, pp. 359-386.

Thomas, J.A., 1983. A quick method for estimating butterfly numbers during surveys. Biol. Conserv. 27, 195-211.

Thomas, J.A., 1984. The conservation of butterflies in temperate countries: past efforts and lessons for the future. In: Vane-Wright, R.I., Ackery, P.R. (Eds.), The Biology of Butterflies, 11th Symposium of the Royal Entomological Society of London. Academic Press, London, pp. 333-353.

Thomas, J.A., Bourn, N.A.D., Clarke, R.T., Stewart, K.E., Simcox, D.J., Pearman, G.S., et al., 2001. The quality and isolation of habitat patches both determine where butterflies persist in fragmented landscapes. Proc. R. Soc: London B 268, 1791-1796.

Waeyenbergh, M., Baguette, M., 1996. First Belgian record of Cotesia vestalis (Haliday, 1834) (Hymenoptera, Braconidae), parasite of Proclossiana eunomia (Esper, 1799) (Lepidoptera, Nymphalidae), new host species. Belg. J. Zool. 126, 79-81.

Warren, M.S., 1992. Butterfly populations. In: Dennis, R.L.H. (Ed.), The Ecology of Butterflies in Britain. Oxford University Press, Oxford, pp. 73-92.

Watkinson, A.R., Sutherland, W.J., 1995. Sources, sinks and pseudo-sinks. J. Anim. Ecol. 64, 126-130.

Wilcove, D.S., McLellan, C.H., Dobson, A.P., 1986. Habitat fragmentation in the temperate zone. In: Soulé, M.E. (Ed.), Conservation Biology: The Science of Scarcity and Diversity. Sinauer Associates, Sunderland, MA, pp. 237-256.

Wilcox, B.A., Murphy, D.D., 1985. Conservation strategy: the effects of fragmentation on extinction. Am. Nat. 125, 879-887. 